\title{
AUTOCYBERBULLYING NA ADOLESCÊNCIA: EMOÇÕES, REPROVAÇÃO ESCOLAR E OUTROS FATORES ASSOCIADOS
}

\author{
Self-Cyberbullying among Adolescents: characteristics, associated emotions and scholastic \\ failure
}

Maria Felícia Henriques Figueiredo - Universidade Coimbra/Portugal Armanda Pinto da Mota Matos - Universidade de Coimbra/Portugal

\begin{abstract}
RESUMO: O autocyberbullying traduz uma forma de autoagressão premeditada e anónima, concretizada predominantemente nas redes sociais, nos jogos online e SMS. As vítimas autoagressivas procuram, sobretudo, aliviar estados de angústia, raiva e frustração, alertar para situações de vitimização por qualquer forma de bullying ou conquistarem atenção. A prevalência desta forma de autoagressão digital entre os adolescentes portugueses permanece desconhecida. Este estudo exploratório, transversal, descritivo e quantitativo teve como objetivo conhecer a prevalência do autocyberbullying, a sua relação com algumas variáveis sociodemográficas e com a reprovação escolar. Participaram 914 adolescentes do $3^{\circ}$ ciclo, sendo 50,3\% rapazes. Foi usado o Questionário Perceção dos Alunos sobre Autocyberbullying, aplicado online. Realizaram-se análises estatísticas, descritivas e inferenciais. Como principais resultados destacam-se a prevalência de $7,4 \%$, sendo $68,7 \%$ rapazes e a correlação significativa entre a reprovação e autocyberbullying. Verificou-se que, no grupo de autocyberbullies, os alunos que já tinham reprovado apresentavam uma média de frequência do comportamento autocyberbullying, superior à dos que nunca tinham reprovado. Evidencia-se que o malestar que este comportamento proporciona requer a atenção dos profissionais da saúde, da educação e dos pais, a definição de políticas conjuntas de educação e saúde e uma intervenção que previna este comportamento nos adolescentes. Sugere-se que combater o insucesso pode constituir uma estratégia de intervenção secundária que vise prevenir o autocyberbullying. É importante retomar o tema em estudos futuros de caráter mais amplo.
\end{abstract}

Palavras-chave: Autocyberbullying. Adolescência. Autoagressão digital. Reprovação acadêmica.

ABSTRACT: resumo no idioma inglês. Self-cyberbullying reflects a form of premeditated and anonymous self-aggression which takes place predominantly on social networks, in online games and by SMS. Self-aggressive victims attempt, above all, to alleviate states of anguish, rage and frustration, to warn of situations of victimisation due to other forms of bullying or to seek attention. The prevalence of this form of digital selfaggression among Portuguese adolescents continues to be unknown. This exploratory, transversal, descriptive and quantitative study aims to examine the prevalence of selfcyberbullying, its relationship with some social and demographic variables and links with scholastic failure. This study involved the participation of 914 adolescents studying in 3rd cycle education, of which $50.3 \%$ were male. It used an online questionnaire entitled "Student Perceptions of Self-Cyberbullying". Statistical, descriptive and inferential analyses were carried out. Key results include a prevalence rate of $7.4 \%$, of which $68.7 \%$ were boys and there was a significant relationship between self-cyberbullying and scholastic failure. It was evident that, within the group of self-cyberbullies, students who 
had already failed had a higher average rate of self-cyberbullying behaviour than those who had never failed. It is clear that the affliction caused by this behaviour requires the attention of health and education professionals and parents. It also requires the definition of combined education and health policies and intervention to prevent against such behaviour in youths. It suggests that combating scholastic failure could be a secondary intervention strategy aimed at preventing self-cyberbullying. It is important to shed light on this subject in more comprehensive studies in the future.

Keywords: Autocyberbullying. Adolescence. Digital aggression. School failure.

\section{INTRODUÇÃO}

O comportamento agressivo compreende a agressão dirigida a alguém ou ao próprio (CARMO, 2013). A forma autodirigida, com ou sem intencionalidade, é comumente designada por autoagressão ou self-harm (BOYD, 2010; ENGLANDER, 2012).

De acordo com vários autores (BANDURA, 1977; KRISTENSEN et al., 2003; BORGES, 2012), diferenciam-se dois tipos de motivos ou de funções do comportamento agressivo: 1- reativos, normalmente associados a comportamentos defensivos, a retaliação a situações percecionadas como provocações, hostilidades e ameaças ou a situações de frustração; 2- proativos ou instrumentais, quando visam atingir a concretização de um objetivo, podendo ou não causar dano à vítima. Neste último caso, é premeditadamente antecipado o resultado que serve o objetivo do agressor: conquistar atenção, promover culpa nos outros, obter reforço positivo, exercer poder sobre outros (familiares, amigos ou pares), expressar a raiva e hostilidade sentidas ou reduzir estados de tensão (BAUMAN et al., 2013; GUERREIRO, 2014; FARIA, 2015).

No que se refere à autoagressão, alguns autores (REIS et al., 2012; CALVETE et $a l ., 2015)$ consideram que a mesma induz a libertação de endorfinas responsáveis pela produção de uma sensação de bem-estar, ainda que transitória, pela diminuição da ansiedade, angústia e tristeza. Ou seja, regista-se uma diminuição das emoções negativas e um aumento de sentimentos positivos e sensação de alívio em resultado da autoagressão.

Este reforço emocional (diminuição das emoções negativas) pode assumir caráter aditivo, instalar-se uma lógica de regresso sistemático a esta estratégia, quando sentimentos de insatisfação ou os resultados que se deseja não se concretizam (KRISTENSEN et al., 2003; ENGLANDER, 2012), para além de poder funcionar como

Educação, Psicologia e Interfaces, Volume 2, Número 2, p. 57-76, Maio/Agosto, 2018. 
catalisador para outros comportamentos de risco, designadamente o suicídio (BAUMAN et al., 2013; GUERREIRO, 2014; FARIA, 2015).

O comportamento autoagressivo tem vindo a ser relacionado com diversos fatores, nomeadamente o uso indevido das tecnologias digitais, experiências negativas na Internet, o cyberbullying (BOYD, 2010; ENGLANDER, 2012; GUERREIRO, 2014; PATCHIN, HINDUJA, 2017), a baixa autoestima, a rejeição dos pares, fatores sociodemográficos (Guerreiro, 2014), o conhecimento de que outros se autoagridem (ROCHA, 2015), problemas escolares, designadamente a ideia de que no domínio académico os resultados alcançados são inferiores ao esperado, a dificuldades de aprendizagem e a um baixo QI (FISHER et al., 2012; GUERREIRO, 2014; SELFHARM UK, 2015).

Muitos adolescentes convivem frequentemente com o insucesso escolar e com a comparação social do seu desempenho, face aos pares, sendo este insucesso penalizante, uma vez que o adolescente deixa de estar em igualdade com os demais.

A experiência da reprovação pode promover um menor autoconceito académico (VAZ SERRA, 1988), uma tendência para o sujeito se considerar inferior ou incapaz, para desenvolver sentimentos de insegurança, de menos valia e de vergonha e uma personalidade autocritica (GILBERT et al., 2004; CASTILHO et al., 2010).

Tomar consciência do insucesso, ou da perda de valor na relação com os outros, pode favorecer sentimentos de vergonha e autocrítica, os quais podem promover um estado de perseguição interna conducente à vontade de se vingarem dos seus próprios fracassos (GILBERT, 2005).

O medo e a vergonha podem também afetar a capacidade de os adolescentes expressarem sentimentos, levando-os a subordinarem-se à própria autocrítica, a evitarem pedidos de ajuda ou o apoio de outras pessoas, em detrimento de outras estratégias, tais como a autoagressão (CASTILHO et al., 2010).

Num contexto de uso generalizado das tecnologias digitais pelos adolescentes, têm-se registado uma nova forma de agressão dirigida ao próprio, designada de digital self-harm, münchausen digital (FELDMAN, 2000; ENGLANDER, 2012; PATCHIN, HINDUJA, 2017) ou autocyberbullying, por serem perpetradas recorrendo aos media digitais.

Trata-se de um comportamento praticado de forma deliberada e anónima por um indivíduo, que consiste em provocar dano psicológico a si próprio recorrendo à simulação 
de cyberbullying: publicação, partilha de conteúdos prejudiciais ou envio de mensagens hostis sobre si a alguém, através das redes sociais (RSI), chats ou jogos online. Com este comportamento, os adolescentes pretendem conquistar a atenção e a admiração dos seguidores online (pais, amigos ou pares) e, desse modo, levá-los a preocuparem-se mais consigo. Pretende ainda aliviar estados de raiva, frustração e angústia sentidas, verem publicados elogios sobre si ou culparem alguém de uma agressão, entre outras.

A literatura (ENGLANDER, 2012; PATCHIN, HINDUJA, 2017) confirma a presença da problemática na adolescência, aponta uma prevalência situada entre $6 \%$ (PATCHIN; HINDUJA, 2017) e 10\% (ENGLANDER, 2012) e destaca a importância de se conhecer e compreender possíveis fatores favorecedores, designadamente fatores escolares, como a reprovação, e fatores sociodemográficos.

Assim, o estudo teve como objetivo conhecer a prevalência na adolescência, pesquisar a relação entre a reprovação escolar e o autocyberbullying bem como identificar outros fatores associados ao comportamento. $\mathrm{O}$ artigo apresenta alguns resultados que integram um estudo mais amplo, desenvolvido no âmbito de um projeto de investigação da tese de doutoramento intitulada "Autocyberbullying: prevalência, motivações e impacto entre alunos do $3^{\circ}$ ciclo do ensino básico do concelho de Leiria”.

\section{MATERIAL E MÉTODO}

Trata-se de um estudo não experimental, de carácter exploratório, já que pretendeu estudar uma realidade desconhecida entre adolescentes portugueses, retrospetivo (teve em conta a prática do autocyberbullying nos últimos seis meses), transversal (a medição das variáveis foi realizada num único momento) e correlacional (o objetivo foi explorar relações entre variáveis e identificar possíveis fatores associados ao comportamento (MARÔCO, 2014).

\subsection{Instrumento}

Os dados foram colhidos em 2016, recorrendo a um questionário aplicado online - Questionário Perceções dos Alunos sobre Autocyberbullying (QPAA), concebido para a investigação anteriormente referida, que se encontra estruturado em 6 partes: 1- Dados pessoais; 2- Posse e uso das tecnologias; 3- Prevalência do bullying e do cyberbullying; 4- Prevalência e caracterização geral do autocyberbullying; 5-Família e escola; 6- 
Medidas de personalidade e atitudes psicossociais (Escala de Autoconceito, Autoestima e Autocriticismo).

A medição da prevalência do autocyberbullying foi efetuada através de 3 questões: a primeira, consiste numa pergunta geral sobre se alguma vez praticaram autocyberbullying (formato Sim/não); a segunda é composta por nove itens que apresentam comportamentos específicos de autocyberbullying (formato likert de 5 pontos de $1=$ nunca a $5=$ várias vezes por semana); a terceira mede o conhecimento de casos, ou seja, se os participantes têm um colega/amigo autocyberbullie (formato $\operatorname{Sim} /$ não).

Esta medição baseou-se num ponto de corte: nunca ter praticado (nunca) e ter praticado pelo menos uma vez um comportamento ( $\geq$ uma vez).

A variável reprovação académica foi medida através de uma questão, composta por dois itens: "Já reprovaste alguma vez?", apresentada em formato dicotómico Sim/Não) e, se sim, “Quantas vezes?” - Escreve o número.

\subsection{Procedimentos de recolha e análise de dados}

Confirmado o interesse das 10 escolas públicas /agrupamentos com $3^{\circ}$ ciclo do concelho de Leiria em colaborarem no estudo, obtida a autorização para aplicação do questionário em meio escolar da Direção-Geral da Educação -DGE, através do sistema de Monitorização de Inquéritos em Meio Escolar - MIME (inquérito no 27100013) e da Comissão Nacional de Proteção de Dados - CNPD e de recolhidos os consentimentos informados dos encarregados de educação, foi enviado a cada escola (via email formal e entregue na direção da escola em suporte de papel) o link de acesso ao questionário online, os procedimentos e condições a respeitar antes, durante o acesso e no preenchimento do mesmo.

O preenchimento decorreu em ambiente escolar, durante uma aula, sob a orientação de um/a professor/a. Toda a turma acedeu ao mesmo tempo e cada participante respondeu individualmente, e uma só vez, ao questionário cujo preenchimento demorou entre 20 e 30 minutos.

Foi garantido o anonimato das respostas dos participantes e o direito à nãoparticipação, através do Consentimento Livre e Esclarecido integrado no corpo do questionário. A taxa de resposta foi de $36 \%$. 
$\mathrm{Na}$ análise dos dados usou-se o SPSS $_{\mathrm{v} 21}$ e um nível de significância 0,05. A fim de analisar a relação entre idade, sexo, reprovação e autocyberbullying utilizou-se o teste $\chi^{2}$, a análise correlacional e de regressão linear, teste Post Hoc e $t$ de Student e ANOVA. A variável nominal sexo foi transformada em variável dummy para efeito de análise correlacional.

\subsection{Amostra}

A amostra, não probabilística, resultou das respostas de 914 alunos do $3^{\circ}$ ciclo ( $7^{\circ}, 8^{\circ}$ e $9^{\circ}$ anos) que submeteram o seu questionário online completo, sendo $49,7 \%$ $(\mathrm{n}=454)$ raparigas e $50,3 \%$ rapazes $(\mathrm{n}=460)($ Tabela 1$)$.

As idades dos participantes variavam entre 11 e 18 anos (rapazes: $\bar{x}=13,56$, $\mathrm{DP}=1,23$; raparigas: $\bar{x}=13,37, \mathrm{DP}=1,05)$.

Tabela 1: Caracterização da amostra por sexo, idade, ano de escolaridade e experiência de reprovação

\begin{tabular}{|c|c|c|c|c|c|c|c|c|c|}
\hline \multirow{3}{*}{ Sexo } & \multicolumn{3}{|c|}{$(n ; \%)$} & \multicolumn{3}{|c|}{$(n ; \%)$} & $\mathbf{N}$ & Teste $\chi^{2}$ & $p$ \\
\hline & \multicolumn{3}{|c|}{$\begin{array}{c}\text { Rapazes } \\
(460 ; 50,3)\end{array}$} & \multicolumn{3}{|c|}{$\begin{array}{l}\text { Raparigas } \\
(454 ; 49,7)\end{array}$} & 914 & 0,039 & 0,843 \\
\hline & $7^{\circ}$ & $8^{\circ}$ & $9^{\circ}$ & $7^{\circ}$ & $8^{\circ}$ & $9^{\circ}$ & & & \\
\hline \multirow[t]{2}{*}{ Ano } & $(163 ; 28,4)$ & $(206 ; 22,5)$ & $(91 ; 9,6)$ & $(144 ; 15,5)$ & $(215 ; 23,5)$ & $(95 ; 10,5)$ & 914 & 1,45 & 0,493 \\
\hline & $\bar{x}$ & \multicolumn{2}{|c|}{$D P$} & $\bar{x}$ & $D P$ & & & Teste $t$ & $p$ \\
\hline Idade & 13,56 & \multicolumn{2}{|c|}{1,23} & $13, .37$ & 1,05 & & 914 & 2,55 & $<0,05$ \\
\hline Reprovação & 1,39 & \multicolumn{2}{|c|}{0,61} & 1,29 & 0,58 & & 195 & 1,083 & 0,280 \\
\hline
\end{tabular}

Apresentavam pelo menos uma reprovação no percurso escolar 21,3\% $(n=195)$ dos participantes. Entre os que reprovaram não se registaram diferenças significativas $[t$ $(194)=1,083 ; p=0,280]$ no número médio de reprovações dos rapazes (rapazes: $\bar{x}=1,39$; $\mathrm{DP}=0,61$; raparigas) e das raparigas $(\bar{x}=1,29 ; \mathrm{DP}=0,58)$.

\section{RESULTADOS E DISCUSSÃO}

A prevalência do autocyberbullying registada na amostra foi de 7,4\% ( $\mathrm{n}=67)$, sendo $68,7 \%$ rapazes. Conheciam ou tinham um amigo autocyberbullie $11 \%$ dos participantes (Erro! Fonte de referência não encontrada.).

Educação, Psicologia e Interfaces, Volume 2, Número 2, p. 57-76, Maio/Agosto, 2018.

ISSN: 2594-5343. DOI: https://doi.org/10.37444/issn-2594-5343.v2i2.101 
Autocyberbullying na adolescência: emoções, reprovação escolar e outros fatores associados

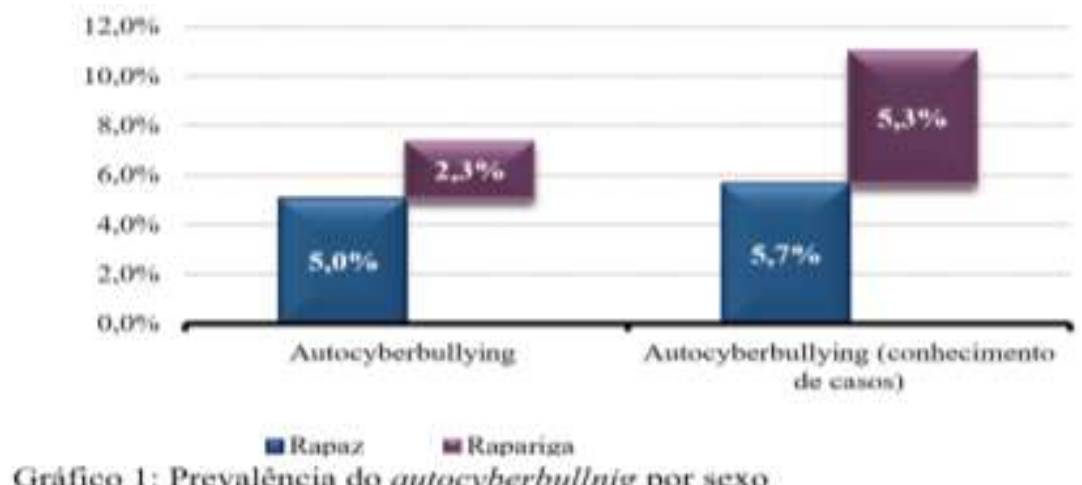

Gráfico 1: Prevaléneia do autoçberbullnig por sexo

Os dados mostram que praticar autocyberbullying não é independente do sexo [ $\left.c^{2}(1)=9,775 ; p<0,05\right]$, uma vez que a média registada nos rapazes foi significativamente superior $\left[t(911)=3,140 ; p<0,05 ; \pi=0,88 ; \iota^{2}=0,011\right]$ à das raparigas.

A variável sexo (Tabela 2) mostrou estar negativa e significativamente $[r(910)=$ $\left.-0,076 ; p<0,05 ; i^{2}=0,006 ; \pi=0,83\right]$ correlacionada com o autocyberbullying, corroborando outros estudos empíricos (ENGLANDER, 2012; PATCHIN, HINDUJA, 2017) sobre autocyberbullying que defendem que os rapazes apresentam atitudes mais favoráveis ao comportamento.

Tabela 2: Teste $t$ e correlação de Pearson entre autocyberbullying e sexo

\begin{tabular}{|c|c|c|c|c|c|c|c|c|c|c|c|}
\hline \multirow[b]{2}{*}{ Sexo } & \multirow[b]{2}{*}{$N$} & \multirow[b]{2}{*}{$\overline{\mathrm{x}}$} & \multirow[b]{2}{*}{$D P$} & \multicolumn{5}{|c|}{ Teste $t$} & \multicolumn{3}{|c|}{ Correlação de Pearson } \\
\hline & & & & $g l$ & $t$ & $p$ & $i^{2}$ & $\pi$ & $r$ & $p \quad i^{2}$ & $\pi$ \\
\hline $\lg \begin{array}{l}\hat{O} \\
q\end{array}$ & $\begin{array}{l}458 \\
453\end{array}$ & $\begin{array}{l}1,10 \\
1,05\end{array}$ & $\begin{array}{l}0.30 \\
0,21\end{array}$ & 911 & 3,140 & $<0,05$ & 0,011 & 0,880 & $-0.076^{*}$ & $<0,050,01$ & 0,8 \\
\hline
\end{tabular}

${ }^{*}$ Correlação significativa ao nível 0,$05 ; \iota^{2}=$ dimensão do efeito da variável idade; $\pi$ potência do teste.

Neste estudo, os rapazes foram os que mais se autoagrediram online o que pode justificar-se pelo facto de, segundo o estado da arte (ENGLANDER, 2012; PATCHIN, HINDUJA, 2017), sendo mais propensos a sentirem raiva, poderem também sentir-se mais instigados à agressão, enquanto as raparigas se apresentam como mais capazes de compreender as suas emoções.

Não se registaram diferenças significativas $\left[t(909)=0,947, p>0,05 ;{ }^{2}{ }^{2}=0,020\right.$, $\pi=0,895]$ na média de idades (Tabela 2$)$ dos que nunca praticaram $(13,46 ; \mathrm{DP}=1,14)$ e a dos que praticaram $(13,60 ; \mathrm{DP}=1,32)$ pelo menos uma vez autocyberbullying. 
As redes sociais (21\%), os jogos online (18\%) e os SMS/MMS (18\%) foram os contextos de maior ocorrência desta forma de agressão, a qual, independentemente do sexo $\left[\chi^{2}(3)=5,533 ; p=0,137\right]$, foi praticada (Gráfico 1) sobretudo em casa $(31,3 \%)$, na escola $(13,4 \%)$ ou noutro local $(40,3 \%)$, recorrendo a computadores $(53 \%)$, smartphones $(42 \%)$ e tablets $(5,3 \%)$.

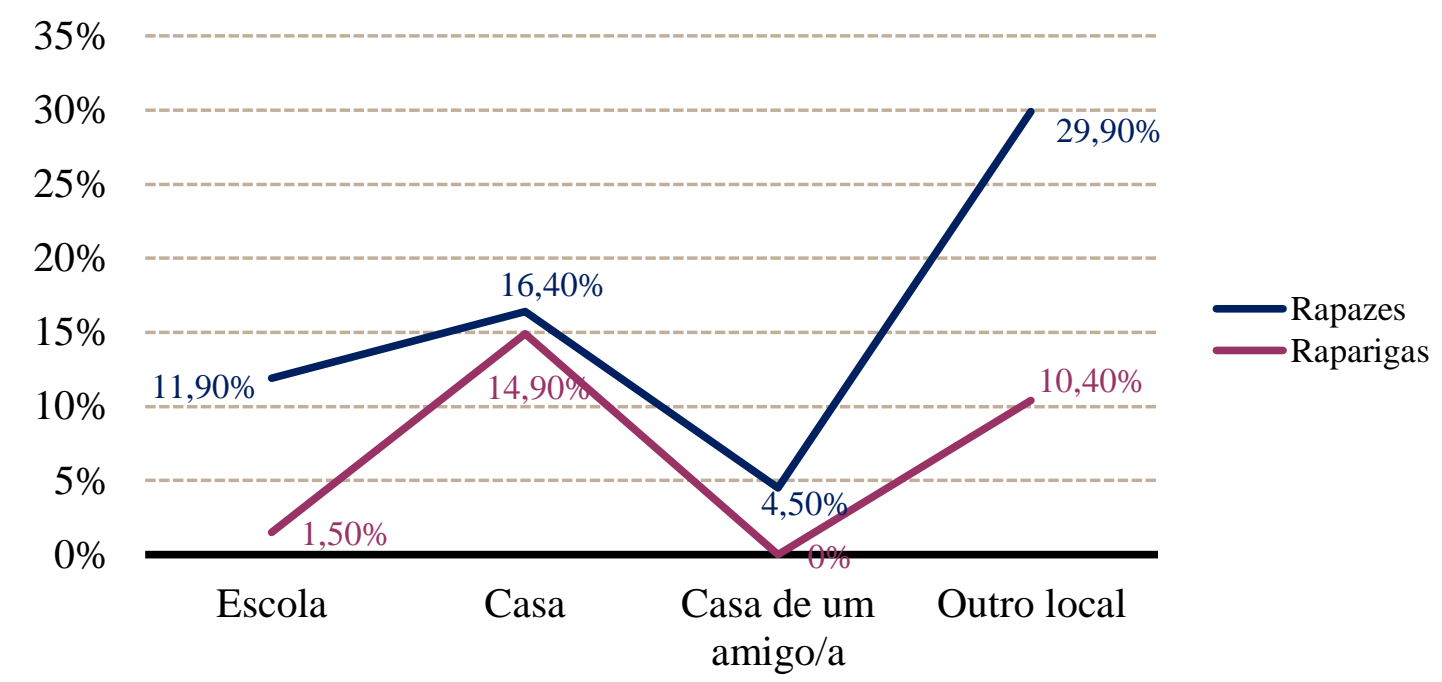

Gráfico 1:Local da prática de autocyberbullying por sexo

A escolha da escola e da própria casa releva a importância que os cuidadores/pais e os professores podem assumir enquanto agentes na prevenção e na sensibilização para a problemática.

Aliviar a raiva $(23,9 \%)$, a frustração $(20,9 \%)$ e a angústia (11\%), pedir ajuda $(11,9 \%)$, alertar para vitimização por bullying $(19,4 \%)$ ou cyberbullying $(6 \%)$, estar descontente com o próprio (10,4\%), retaliar ou culpar alguém de uma agressão (9\%) ou divertir-se $(16,4 \%)$ foram algumas motivações apontadas; tristeza $(44.3 \%)$, angústia $(9 \%)$, vergonha $(37,7 \%)$, medo $(32,8 \%)$, ansiedade $(31,1 \%)$, sentir-se pior do que antes $(36,1 \%)$, sem vontade de ir à escola $(37 \%)$ ou com vontade de morrer $(8,9 \%)$ foram algumas emoções experienciadas; ser gozado/a na escola $(10,4 \%)$, vítima de cyberbullying $(7,4 \%)$ ou piorar os resultados escolares $(14,9 \%)$ forma consequências pessoais sociais e académicas apontadas e promovidas pelo comportamento.

Os resultados apresentados mostram uma prevalência que se situa dentro da moldura de valores relatados no estado da arte, 6\% e 10\% (ENGLANDER, 2012; PATCHIN, HINDUJA, 2017), e próxima da registada na autoagressão não mediada pelas tecnologias (7,4\% e 13,8\%), registada na adolescência (RISSANEN et al., 2011;

Educação, Psicologia e Interfaces, Volume 2, Número 2, p. 57-76, Maio/Agosto, 2018. 
RASMUSSEN, HAWTON, 2014; CALVETE et al., 2015), designadamente em Portugal (7\% e 35\%) (GUERREIRO, 2014).

Também os resultados deste estudo, relativamente aos meios, aos contextos de ocorrência e às emoções promovidas, são coerentes com as conclusões reportadas na literatura (ENGLANDER, 2012; PATCHIN, HINDUJA, 2017).

Ainda que o autocyberbullying possa incluir ações inofensivas, outras são inequivocamente intimidatórias. Trata-se de encenar um esquema comunicativo que envolve o envio de mensagens contendo informações pessoais, ofensivas, humilhantes ou comprometedoras sobre si a alguém, através das redes sociais, das plataformas dos jogos online, fóruns, Websites, blogues ou SMS/MMS, seguido de uma resposta às mesmas, realizada pelo próprio, às quais podem suceder-se mensagens positivas ou então críticas e outras formas de agressão psicológica (cyberbullying).

Recorrer à simulação de self-cyberbullying possibilita ao autocyberbullie enquadramentos através dos quais, pessoal ou socialmente, pode beneficiar, atingindo o objetivo que determinou o comportamento.

Pela análise dos comportamentos, registados neste estudo, constantes no Gráfico 2 verifica-se que este ato hostil, praticado por um indivíduo deliberada e anonimamente, pode assumir as seguintes tipologias:1) Simulação - encenar self-cyberbullying com o objetivo de criar a ilusão/chamar a atenção para vitimização; 2) Dissimulação- fazer de conta que é outra pessoa para publicar informação sensível sobre o próprio; 3) Manifestar ódio - ameaçar-se a si próprio; 4) Exclusão - fingir ter sido banido de um grupo numa rede social, fórum, blogue ou outro; 5) Engano - convencer alguém, sem que esta se aperceba, revelar informações embaraçosas sobre si, partilhando-as online; 6) Denegrição - publicar informações hostis sobre o próprio; 7) Assédio - envio de mensagens ofensivas sobre si a alguém; 8) Divulgar - Revelar informações pessoais com o objetivo de serem utilizadas por outros para o/a insultarem. 
No Gráfico 2 apresenta-se ainda a frequência dos comportamentos autocyberbullying, distribuídos segundo o sexo.

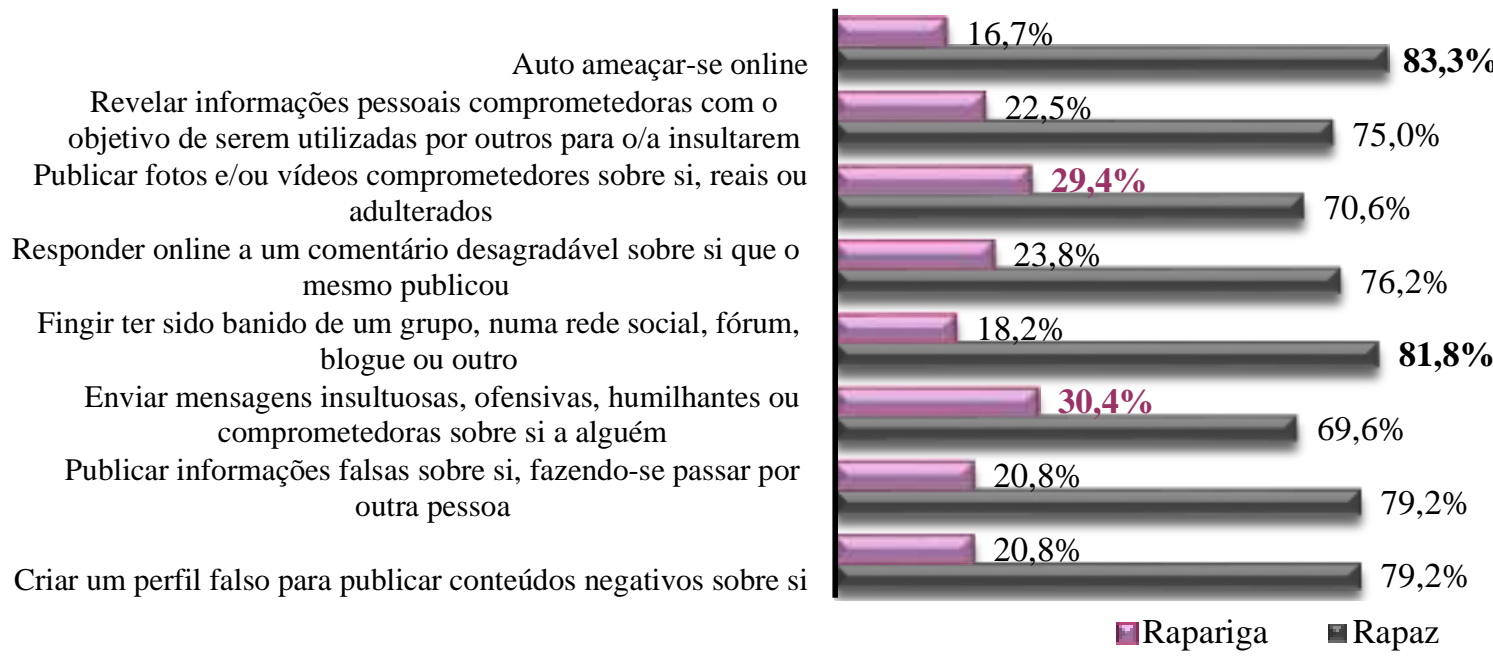

Gráfico 2: Frequência dos comportamentos autocyberbullying por sexo

Tendo em conta as respostas dos participantes, o autocyberbullying tem inerente os seguintes motivos ou funções: 1- medida de coping para fazer face a um estado de debilidade emocional nomeadamente, aliviar o estado de raiva $(23,9 \%)$, de frustração $(20,9 \%)$ e de angústia (11,9\%) sentidos ou estar descontente com o próprio $(10,4 \%)$, sendo este último significativamente mais evidente nos rapazes $\left[c^{2}(1)=5,837 ; p<0,05\right]$; 2- pedido de ajuda (11,9\%) ou alerta para situações de vitimização por bullying $(19,4 \%)$ e cyberbullying (6\%) de que foram/estavam a ser alvo; 3- motivos hedonistas tais como brincadeira (16,4\%), retaliar ou culpar alguém de uma agressão (9\%); 4- motivos egóicos ou de autoafirmação, tais como ver publicadas elogios/mensagens positivas sobre si (9\%) ou testar se conseguiam suportar comentários negativos sobre si (4,5\%); 5- autopunição $(4,5 \%)$ por algo que fizeram $(4,5 \%)$ e causar mal-estar ao próprio $(6 \%)$; 6- motivos de afiliação, designadamente conquistar atenção de amigos/as (9\%), a confiança de pessoas na Internet $(6 \%)$ ou levar a família a preocupar-se mais com eles/elas. De um modo geral, as funções referidas encontram-se descritas no estado da arte (ENGLANDER, 2012; PATCHIN; HINDUJA, 2017).

Praticar autocyberbullying promoveu nos praticantes emoções negativas primárias (DAMÁSIO, 2000; EKMAN, 2004) tais como tristeza (44,3\%), angústia (9\%), vergonha $(37,7 \%)$, medo $(32,8 \%)$, ansiedade $(31,1 \%)$ ou sentir-se pior do que antes $(36,1 \%)$, que encontram apoio em estudos realizados com adolescentes (HAWTON et al.,

Educação, Psicologia e Interfaces, Volume 2, Número 2, p. 57-76, Maio/Agosto, 2018.

ISSN: 2594-5343. DOI: https://doi.org/10.37444/issn-2594-5343.v2i2.101 
2002; PERREN et al., 2010; NUNES, 2012; GUERREIRO, 2014; MATOs et al., 2014) que referem que as vítimas de agressão apresentam frequentemente sentimentos de vulnerabilidade, medo e vergonha.

O autocyberbullying propiciou ainda experiências emocionais extremistas, tais como a vontade de morrer (8,9\%). A ideação suicida e o suicídio estão descritos (BOYD, 2010; SELFHARM UK, 2015) como situações que podem ocorrer no autocyberbullying.

Foi também apontada a falta de vontade de ir à escola $(37,7 \%)$, a que poderá não ser alheio o fato de as publicações efetuadas pelo autocyberbullie permanecerem indefinidamente no espaço virtual e de, nesse sentido, este poder sentir vergonha e medo de ir à escola, pelo receio de ser alvo de cyberbullying (BOYD, 2010; ENGLANDER, 2012; SELFHARM UK, 2015).

Outras emoções descritas, positivas ou neutras, tais como satisfação $(40,3 \%)$, orgulho $(27,9 \%)$, sentir-se inspirado(a), bem e com poder $(27,9 \%)$, remetem para um sentido egóico do comportamento. Também estas emoções surgem relatadas na literatura sobre o autocyberbullying (ENGLANDER, 2012) e sobre cyberbullying (CAETANO et al., 2016) que defende que os agressores manifestam frequentemente emoções de orgulho, indiferença e felicidade após a prática destes comportamentos, para além de destacar a fraca relação entre bullying e a presença de emoções morais, tanto nas vítimas como nos agressores (FRANCISCO et al., 2015), sendo esta dualidade de papéis uma característica presente no autocyberbullying.

Foram ainda descritas emoções que apelam a sentimentos de justiça e a uma necessidade de vingança $(31,1 \%)$, as quais recebem o apoio da literatura (BOYD, 2010; ENGLANDER, 2012; PATCHIN, HINDUJA, 2017) que refere o recurso a esta forma de agressão, impulsiva e destrutiva, como estratégia para lidar com os estados de frustração, ansiedade e sentimentos de culpa ou como uma forma de passar para um estado de maior tranquilidade (PATCHIN, HINDUJA, 2017). A literatura sugere, ainda, que o autocyberbullying representa um prolongamento do cyberbullying (BOYD, 2010; ENGLANDER, 2012; PATCHIN, HINDUJA, 2017).

Qualquer interação social pressupõe a representação e interpretação de uma informação, a análise causal de um acontecimento, a inferência sobre a intenção dos outros, a definição de um objetivo e a construção de uma resposta para a situação e a avaliação da resposta (HABERMAS, 1987; CRICK, DODGE, 1994; HABERMAS, 2012). 
Recorrendo a um modelo de decisão, os sujeitos analisam formas e meios que lhes permitam repor a justiça face a uma situação. Seja por défice de processamento da informação (DAMÁSIO, 2000; EKMAN, 2004), pela atribuição de uma intencionalidade negativa ao outro ou por uma perceção incorreta de um estímulo, predispõem-se à prática de comportamentos hostis e a respostas agressivas dirigidas ao outro.

A vitimização está correlacionada de forma positiva com sintomas de ansiedade e vergonha interna e externa e com experiências de vergonha, reconhecidas como uma componente de extrema importância na propensão para a agressão (GILBERT et al., 2004), na tendência para evitar o contacto interpessoal e no refúgio no mundo virtual. Experiências repetidas de vitimização podem aumentar a vergonha interna e tendência para desencadear sentimentos negativos que podem manifestar-se através de reações exageradas, como a necessidade de vingança presente no autocyberbullying.

Ainda no campo das emoções sentidas pelos participantes do estudo, o arrependimento (40,3\%) sugere um sentido de julgamento relativamente à prática de autocyberbullying, o qual pode ser entendido no sentido da compreensão, da gestão das próprias emoções e motivações e num desencorajar da reincidência no comportamento.

Sintetizando, de um modo geral as experiências emocionais assumidas pelos autocyberbullies não apresentaram diferenças significativas entre sexos. Os autocyberbullies tendem a sentir frequentemente tristeza, arrependimento, vergonha, medo, vontade de se vingarem ou sentiram-se pior do que antes. Contudo, é preocupante, o facto de alguns terem mostrado satisfação e vontade de voltar a autoagredir-se.

Saber gerir e usar as emoções são competências emocionais relevantes para lidar com as adversidades da vida. Dependendo da intensidade e do modo como os adolescentes as expressam ou deixam fluir, estas podem levá-los a um desajuste, ou orientá-los num sentido mais adaptativo.

Os resultados deste estudo apontaram implicações do comportamento para os envolvidos (Gráfico 3), que se refletem na escola e nas famílias, donde se destacam, sem diferença significativa entre sexos $(p>0,05)$, os piores resultados escolares obtidos $(14,9 \%)$ e ser gozado/a na escola $(10,4 \%)$. No entanto, para $26,8 \%$, que obtiveram mensagens de apoio, e para 14,9\%, que considerou que a família passou a preocupar-se mais com eles/elas, recorrer a esta estratégia foi entendida como uma medida positiva.

Educação, Psicologia e Interfaces, Volume 2, Número 2, p. 57-76, Maio/Agosto, 2018. 
Os meus pais proibiram-me de ir à Internet Fui vítima de cyberbullying

Outro

Fui gozado(a) na escola

Os meus resultados escolares pioraram

A minha família passou a preocupar-se mais comigo

Recebi elogios e mensagens de apoio na Internet

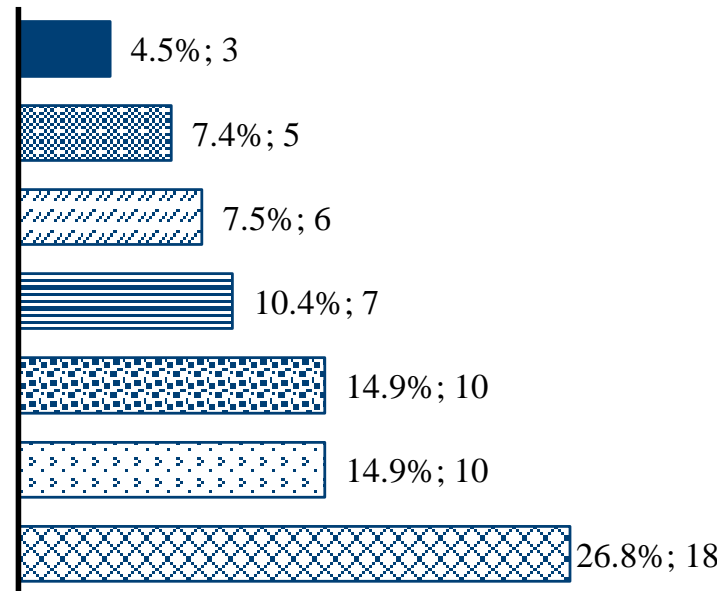

$\begin{array}{llllllll}0 & 3 & 6 & 9 & 12 & 15 & 18 & 21\end{array}$

Gráfico 3: Frequência relativa e absoluta das repercussões do autocyberbullying

Gostar de estudar, e fazê-lo com o mesmo prazer com que se desfruta de outras atividades, é algo que se aprende, mas um ingrediente que um adolescente só conseguirá alcançar se dispuser de bem-estar. A atenção do aluno durante as aulas revela um esforço de concentração e de participação que podem indiciar predisposição para a aprendizagem.

Regra geral, as vítimas de diferentes formas de bullying apresentam alterações na capacidade de concentração e de aprendizagem, perda de memória e insegurança por estarem na escola.

Num aluno que acumule insucessos, rapidamente a ideia de que não vai conseguir ir longe assola a sua mente, comprometendo a sua saúde psicológica e o seu ego. Mesmo que este procure que este sofrimento não seja percetível nas suas atitudes, mais tarde ou mais cedo, os fracassos individuais podem manifestar-se e tornar-se um problema social (FARRINGTON et al., 1990).

Um Q.I abaixo da média, as dificuldades de aprendizagem e um ambiente escolar instável, são apontados como preditores da prática de atos deliberados de autoagressão e de tentativas de suicídio (FARRINGTON et al., 1990).

Os sujeitos que já reprovaram apresentam maior propensão para desenvolverem problemas psicossociais (HINDUJA, PATCHIN, 2010), vivência de vergonha e outros estados emocionais negativos (depressão, ansiedade e stresse) (COSTA, PEREIRA, 2010; BENTO, 2011; GUERREIRO, 2014) favorecedores da prática de cyberbullying (PINTO, 2011) e do autodano (NUNES, 2012). 
A adolescência é um período associado ao neurodesenvolvimento a que se atribui uma vulnerabilidade particular, um período no qual os jovens estão mais suscetíveis a estímulos sociais negativos, tais como o ostracismo e as elevadas expectativas dos outros, e a um risco acrescido de perturbações emocionais (HAWTON et al., 2012).

A reprovação, questão problemática pelos efeitos negativos ao nível da estigmatização, é hoje muito questionada, e encarada como um último recurso.

Os resultados deste estudo (Tabela 3) indicam que os adolescentes que já reprovaram $(\overline{\mathrm{x}}=1,12 ; \mathrm{DP}=0,33)$ apresentaram uma média de comportamentos autocyberbullying significativamente $[\mathrm{t}(909)=-3,029 ; p<0,05]$ superior à dos que nunca $\operatorname{reprovaram}(\overline{\mathrm{x}}=1,06 ; \mathrm{DP}=0,24)$.

Tabela 3: Média de comportamentos autocyberbullying e reprovação escolar

\begin{tabular}{|c|c|c|c|c|c|c|c|}
\hline \multirow{4}{*}{ Autocyberbullying } & \multicolumn{3}{|c|}{ Reprovação } & \multirow[b]{2}{*}{$\mathrm{N}$} & \multicolumn{3}{|c|}{ Teste $t$} \\
\hline & & Sim & Não & & $g l$ & $t$ & $p$ \\
\hline & $n$ & 194 & 717 & 911 & & & \\
\hline & $\overline{\mathrm{x}}$ & 1,12 & 1,06 & & 000 & 3070 & 0003 \\
\hline & DP & 0,33 & 0,24 & & 909 & $-3,0<9$ & 0,005 \\
\hline
\end{tabular}

A reprovação (Tabela ) apresentou-se como uma variável significativamente correlacionada com a perpetração do autocyberbullying $\left[r(914)=0,100 ; p<0,05 ; \imath^{2}=\right.$ $0,01 ; \pi=0,857]$ ou seja, quanto maior o número de reprovações maior é o risco de se ser autocyberbullie.

Tabela 4: Correlação de Pearsan entre autocyberbullying e reprovação escolar

\begin{tabular}{ccccc}
\hline & \multicolumn{4}{c}{ Autocyberbullying } \\
\cline { 2 - 5 } Reprovação & $r$ & $p$ & $i^{2}$ & $\pi$ \\
& $0,100^{* *}$ & 0,003 & 0,01 & 0,85 \\
\hline
\end{tabular}

** Correlação significativa ao nível 0,$01 ; \boldsymbol{i}^{2}=$ dimensão do efeito da variável reprovação; $\pi$ = potência do teste.

A associação positiva encontrada contraria o preconizado na literatura (BOYD, 2010; BOYD et al., 2011; ENGLANDER, 2012) que sugere que o desempenho académico não é um traço do autocyberbullie. Para fundamentar esta conclusão, os investigadores referem que, ainda que os autocyberbullies sejam sujeitos vulneráveis ou impopulares em contexto escolar (BOYD, 2010; BOYD et al., 2011; ENGLANDER, 2012), as situações de suicídio relatadas na comunicação social (e.g. HANNAH SMITH), e que tiveram origem no autocyberbullying, diziam respeito a sujeitos com um percurso académico irrepreensível (WINTERMAN, 2013).

Educação, Psicologia e Interfaces, Volume 2, Número 2, p. 57-76, Maio/Agosto, 2018.

ISSN: 2594-5343. DOI: https://doi.org/10.37444/issn-2594-5343.v2i2.101 
Os resultados obtidos do presente estudo são, no entanto, coerentes com o estado da arte sobre cyberbullying, que defende que as vítimas deste comportamento apresentam. regra geral, resultados escolares inferiores ou um desempenho escolar negativo, e que o número de reprovações está positiva e significativamente associado ao cyberbullying (HINDUJA, PATCHIN, 2010; PINTO, 2011). São também consistentes com o veiculado nos estudos sobre autodano que sustentam existir diferenças estatisticamente significativas entre ter ou não reprovado e o maior ou menor envolvimento nesse comportamento (NUNES, 2012).

Assim, pelos resultados do estudo, consideramos que a reprovação pode favorecer o desencadear do círculo Reprovação $\rightarrow$ Frustração $\rightarrow$ Autocyberbullying $\rightarrow$ Falta de vontade de ir à escola $\rightarrow$ Piores resultados escolares $\rightarrow$ Reprovação, que importa interromper.

Nesse sentido, à semelhança de outros países que apresentam elevada qualidade no ensino, devem ser encontradas mais/novas alternativas que previnam o insucesso escolar do aluno.

\section{CONSIDERAÇÕES FINAIS}

Os resultados apresentados levantam algumas questões em torno da relação entre o autocyberbullying e a reprovação escolar. É possível que, pela prevalência do autocyberbullying e a relação encontrada com a reprovação revele o quanto esta forma de autoagressão psicológica se apresenta ancorada também nesta experiência negativa, pelo que combater o insucesso continua a ser um desafio para a Educação.

Reconhecer a reprovação como uma experiência a evitar remete para a necessidade de novos estudos que analisem o papel de variáveis associadas ao insucesso escolar no autocyberbullying, e o seu impacto segundo o sexo, tendo em conta que nesta investigação se evidenciou que o comportamento não se manifesta igualmente nos rapazes e raparigas.

Intervir, aos primeiros sinais de insucesso, pode constituir uma forma de intervenção secundária que vise prevenir o autocyberbullying. Espera-se que a escola (re)conheça as vivências e dificuldades dos alunos, que seja capaz de atuar eficazmente, combatendo o insucesso, que proporcione informação sobre o autocyberbullying, treino de competências de regulação emocional, que aborde o autoconhecimento e a compreensão de comportamentos assumidos. 
Os contextos de ocorrência e os meios usados na prática de autocyberbullying evidenciam a pertinência da definição de programas dirigidos aos alunos mobilizando, para o efeito, as próprias tecnologias (redes sociais ou outras plataformas) para abordar aspetos relacionados com a problemática.

É importante ressalvar que as conclusões do estudo não podem ser assumidas como representativas da realidade nacional, tendo em conta a natureza exploratória e transversal, o caráter não experimental e o facto de o estudo ter sido realizado numa única área geográfica. Nesse sentido, incentiva-se a realização de novos estudos, com metodologias, participantes e delineamentos diferentes, envolvendo aspetos associados à reprovação e a outras variáveis escolares, que permitam evidenciar fatores favorecedores e protetores do fenómeno.

O autocyberbullying necessita ser perspetivado como uma forma de agressão face à qual, num momento de fragilidade, quaisquer adolescentes se podem tornar vítimas. Importa apoiá-los, para que encontrem mecanismos que lhes permitam transformar emoções negativas em ações valorizadoras.

\section{REFERÊNCIAS BIBLIOGRÁFICAS}

BANDURA, A. Social learning theory. Oxford, England: Prentice-Hall, 1977.

BAUMAN, S.; TOOMEY, R.; WALKER, J. Associations among bullying, cyberbullying, and suicide in high school students. Journal of adolescence, v. 36, n. 2, p. 341-350, 2013.

BENTO, A. O Cyberbullying no Contexto Português. 2011. (Mestrado). Faculdade de Ciências Sociais e Humanas, Universidade da Beira Interior, Covilhã.

BORGES, C. À flor da pele: Algumas reflexões a propósito de um estudo de caso sobre autolesão. 2012. (Mestrado). ISPA - Instituto Universitário das Ciências Psicológicas, Sociais e da Vida

BOYD, D. Digital Self-Harm and Other Acts of Self-Harassment 2010.

BOYD, D.; RYAN, J.; LEAVITT, A. Pro-self-harm and the visibility of youthgenerated problematic content. ISJLP, v. 7, n. 1, p. 1-32, 2011.

CAETANO, A. et al. Emoções no cyberbullying: um estudo com adolescentes portugueses. Educação e Pesquisa, v. 42, n. 1, p. 199-212, 2016. ISSN 1678-4634.

CALVETE, E. et al. Prevalence and functions of non-suicidal self-injury in Spanish adolescents. Psicothema, v. 27, n. 3, p. 223-228, 2015.

Educação, Psicologia e Interfaces, Volume 2, Número 2, p. 57-76, Maio/Agosto, 2018.

ISSN: 2594-5343. DOI: https://doi.org/10.37444/issn-2594-5343.v2i2.101 
CARMO, R. Relações entre Crianças e Jovens em Instituições de Acolhimento. 2013. (Mestrado). Escola Superior de Educação, Instituto Politécnico de Portalegre, Portalegre.

CASTILHO, P.; GOUVEIA, J.; BENTO, E. Auto-criticismo, vergonha interna e dissociação: a sua contribuição para a patoplastia do auto-dano em adolescentes.

Psychologica Coimbra, v. 2, n. 52, p. 332-358, 2010. ISSN 0871-4657. Disponível em:

< Retrived from http://iduc.uc.pt/index.php/psychologica/article/view/1060 >.

COSTA, P.; PEREIRA, B. O bullying na escola: a prevalência e o sucesso escolar. In: UNIVERSIDADE DO MINHO, C., I Seminário Internacional Contributos da Psicologia em Contexto Educativo, 2010, Braga. p.1810-1821.

CRICK, N.; DODGE, R. A review and reformulation of social information-processing mechanisms in children's social adjustment. Psychological Bulletin, v. 115, n. 1, p. 74$101,1994$.

DAMÁSIO, A. O sentimento de Si. Lisboa: Europa América, 2000. ISBN 9789721047570.

EKMAN, P. Qué dice ese gesto? Integral. Barcelona La Magrana, 2004.

ENGLANDER, E. Digital self-harm: Frequency, type, motivations, and outcomes. Massachusetts Aggression Reduction Center. Massachusetts 2012

FARIA, C. Quando a agressão virtual coloca em risco a vida real: Cyberbullying, perceção do suporte social e ideação suicida. 2015. Mestrado (Mestrado). ISPA Instituto Universitário de Ciências Psicológicas, Sociais e da Vida, Lisboa.

FARRINGTON, D.; LOEBER, R.; VAN KAMMEN, W. Long-term criminal outcomes of hyperactivity-impulsivity-attention deficit and conduct problems in childhood. In: ROBINS, L. e RUTTER, M. (Ed.). Straight and devious pathways from childhood to adulthood. New York: Cambridge University Press, 1990. p.62-81.

FELDMAN, M. Munchausen by Internet: detecting factitious illness and crisis on the Internet. Southern medical journal, v. 93, n. 7, p. 669-672, 2000. ISSN 0038-4348.

FISHER, H. et al. Bullying victimisation and risk of self harm in early adolescence: longitudinal cohort study. British Medical Journal, v. 344, p. 1-9, 2012. Disponível em: < http://www.ncbi.nlm.nih.gov/pmc/articles/PMC3339878/pdf/bmj.e2683.pdf >. Acesso em: 12 de fevereiro de 2014.

FRANCISCO, S. et al. Cyberbullying: The hidden side of college students. Computers in Human Behavior, v. 43, p. 167-182, 2015. ISSN 0747-5632.

/GILBERT, P. Compassion and cruelty: A biopsychosocial approach. In: GILBERT, P. (Ed.). Compassion: Conceptualisations, Research and Use in Psychotherapy. Hove, East Sussex: Routledge, 2005. cap. 2, p.9-74. 
GILBERT, P. et al. Criticising and reassuring oneself: An aploration of forms, styles and reasons in female students. British Journal of Clinical Psychology, v. 43, n. 1, p. 31-50, 2004.

GUERREIRO, D. Comportamentos autolesivos em adolescentes:características epidemiológicas e análise de fatores psicopatológicos, temperamento efetivo e estratégias de coping. 2014. (Doutoramento). Faculdade de Medicina, Universidade de Lisboa, Lisboa.

HABERMAS, J. Teoria de la acción comunicativa. Madrid: Taurus, 1987.

HABERMAS, J. Teoria do agir comunicativo: sobre a crítica da razão funcionalista. São Paulo: Martins Fontes, 2012.

HAWTON, K. et al. Deliberate self harm in adolescents: self report survey in schools in England. British Medical Journal, v. 325, n. 7374, p. 1207-1211, 2002. ISSN 09598138. Disponível em: < http://www.bmj.com/content/325/7374/1207 >.

HAWTON, K.; SAUNDERS, K.; O'CONNOR, R. Self-harm and suicide in adolescents. The Lancet, v. 379, n. 9834, p. 2373-2382, 2012. Disponível em: < http://www.thelancet.com/journals/lancet/article/PIIS0140-6736(12)60322-5/abstract $>$. Acesso em: 2015/04/20.

HINDUJA, S.; PATCHIN, J. Bullying, cyberbullying, and suicide. Archives of suicide research, v. 14, n. 3, p. 206-221, 2010. ISSN 1381-1118.

KRISTENSEN, C. et al. Fatores etiológicos da agressão física: Uma revisão teórica. Estudos de Psicologia, v. 8, n. 1, p. 175-184, 2003.

MARÔCO, J. Análise Estatística com o SPSS Statistics. 6. Pero Pinheiro: Report Number, Lda, 2014. ISBN 978-989-96763-4-3.

MATOS, M. et al. A saúde dos adolescentes portugueses em tempos de recessão: dados nacionais 2014. CENTRO DE MALÁRIA E OUTRAS DOENÇAS TROPICAIS. Lisboa 2014.

NUNES, C. Auto-dano e ideação suicida na população adolescente: aferição do questionário de impulso, auto-dano e ideação suicida na adolescência (QIAIS-A). 2012. (Mestrado). Universidade dos Açores, Ponta Delgada.

PATCHIN, J.; HINDUJA, S. Digital Self-Harm Among Adolescents. Journal of Adolescent Health, v. 61, n. 6, p. 761-766, 2017. ISSN 1054-139X. Acesso em: 2017/10/31.

PERREN, S. et al. Bullying in school and cyberspace: Associations with depressive symptoms in Swiss and Australian adolescents. Child and adolescent psychiatry and mental Health Promotion International, v. 4, n. 28, p. 1-10, 2010.

PINTO, T. Cyberbullying: Estudo da prevalência de comportamentos de cyberbullying e sua relação com vivências de vergonha e estados emocionais 
negativos. 2011. (Mestrado). Psicoterapia e Psicologia Clínica, Instituto Superior Miguel Torga, Coimbra.

RASMUSSEN, S.; HAWTON, K. Adolescent self-harm: a school-based study in Northern Ireland. Journal of affective disorders, v. 159, p. 46-52, 2014. ISSN 01650327.

REIS, M.; FIGUEIRA, I.; RAMIRO, L. Jovens e comportamentos de violência autodirigida. Aventura Social: Promoção de Competências e do Capital Social para um Empreendedorismo com Saúde na Escola e na Comunidade. MATOS, M. e TOMÉ, G. Lisboa: Placebo, Editora LDA. 1: 259-276 p. 2012.

RISSANEN, M.; KYLMA, J.; LAUKKANEN, E. A systematic literature review: Selfmutilation among adolescents as a phenomenon and help for it-what kind of knowledge is lacking? Issues in Mental Health Nursing, v. 32, n. 9, p. 575-583, 2011. ISSN 01612840. Disponível em: <

http://search.ebscohost.com/login.aspx?direct=true \&db=a9h\&AN=64486033\&site=eho st-live\&scope $=$ site $>$.

ROCHA, G. Condutas autolesivas: uma leitura pela Teoria do Apego. Revista Brasileira de Psicologia, v. 2, n. 1, p. 62-70, 2015. Disponível em: < http://revpsi.org/wp-content/uploads/2015/04/Rocha-2015-Condutas-autolesivas-umaleitura-pela-Teoria-do-Apego.pdf >. Acesso em: 30/09/2015.

SELFHARM UK. Self-harm statistics. United Kingdom, 2015. Disponível em: < https://www.selfharm.co.uk/get/facts/self-harm_statistics $>$.

VAZ SERRA, A. O auto-conceito. Análise Psicológica, v. 2, n. VI, p. 101-110, 1988. Disponível em: < http://repositorio.ispa.pt/bitstream/10400.12/2204/1/1988_2_101.pdf $>$.

WINTERMAN, D. Cyber self-harm: Why do people troll themselves online? BBC News Magazine. Europa 2013.

\section{Credenciais das autoraes}

FIGUEIREDO, Maria Felícia Henriques. Doutoranda na Faculdade de Psicologia e de Ciências da Educação da Universidade Coimbra/Portugal. E-mail: uc2013109703@student.uc.pt

MATOS, Armanda Pinto da Mota. Faculdade de Psicologia e de Ciências da Educação da Universidade de Coimbra/Portugal.

Endereço para correspondência: Maria Felícia Henriques Figueiredo, contacto: uc2013109703@student.uc.pt

Como citar este artigo (Formato ABNT): FIGUEIREDO, Maria Felícia Henriques de; MATOS, Armanda Pinto da Mota. Autocyberbullying na adolescência: emoções, 
reprovação escolar e outros fatores associados. Educação, Psicologia e Interfaces, v. 2, n. 2, p. 57-76, 2018. DOI: https://doi.org/10.37444/issn-2594-5343.v2i2.101

Recebido: 04/02/2018.

Aceito: 26/07/2018. 